\title{
Frozen Premix Intravenous Piggyback Solution Dosage Form
}

National Cancer Institute

\section{Source}

National Cancer Institute. Frozen Premix Intravenous Piggyback Solution Dosage Form. NCl Thesaurus. Code C68966.

A solution that is reconstituted from a frozen pre-mixed form intended for intravenous administration simultaneously with an established compatible maintenance infusion drug by a mutual intravenous access. 